\title{
Robust Postmortem Survival of Murine Vestibular and Cochlear Stem Cells
}

\author{
Pascal Senn, ${ }^{1,2}$ Kazuo Oshima, ${ }^{1,2}$ Dawn Teo, ${ }^{1,2}$ Christian Grimm, ${ }^{1,2}$ and Stefan Heller ${ }^{1,2}$ \\ ${ }^{1}$ Department of Otolaryngology, Head and Neck Surgery and Molecular and Cellular Physiology, Stanford University School \\ of Medicine, 801 Welch Road, Stanford, CA 94305-5739, USA \\ ${ }^{2}$ Department of Otolaryngology, Head and Neck Surgery, Inselspital, University of Berne, 3010 Berne, Switzerland
}

Received: 19 September 2006; Accepted: 10 February 2007; Online publication: 3 March 2007

\begin{abstract}
Potential treatment strategies of neurodegenerative and other diseases with stem cells derived from nonembryonic tissues are much less subjected to ethical criticism than embryonic stem cell-based approaches. Here we report the isolation of inner ear stem cells, which may be useful in cell replacement therapies for hearing loss, after protracted postmortem intervals. We found that neonatal murine inner ear tissues, including vestibular and cochlear sensory epithelia, display remarkably robust cellular survival, even 10 days postmortem. Similarly, isolation of sphere-forming stem cells was possible up to 10 days postmortem. We detected no difference in the proliferation and differentiation potential between stem cells isolated directly after death and up to 5 days postmortem. At longer postmortem intervals, we observed that the potency of sphere-derived cells to spontaneously differentiate into mature cell types diminishes prior to the cells losing their potential for self-renewal. Three-week-old mice also displayed sphere-forming stem cells in all inner ear tissues investigated up to 5 days postmortem. In summary, our results demonstrate that postmortem murine inner ear tissue is suited for isolation of stem cells.
\end{abstract}

Keywords: utricle, hair cell, regeneration, spiral ganglion, neurosphere

Correspondence to: Stefan Heller - Department of Otolaryngology, Head and Neck Surgery and Molecular and Cellular Physiology • Stanford University School of Medicine - 801 Welch Road, Stanford, CA 94305-5739, USA. Telephone: +1-650-7248086; fax: +1-650-7258502; email: hellers@stanford.edu

\section{INTRODUCTION}

The inability of the cochlea to replace lost hair cells is the major reason for the irreversibility of hearing loss in mammals. A starting point for a potential cure for hearing loss would be to regenerate hair cells either by transplantation of replacement cells or by stimulating cells of the damaged inner ear sensory epithelia to proliferate and to differentiate into hair cells.

Cells with the capacity to proliferate and to differentiate into hair cells and other cell types have been isolated from the murine vestibular system ( $\mathrm{Li}$ et al. 2003a) and, more recently, from cochlear tissues such as the organ of Corti and the spiral ganglion (Oshima et al. 2007). These proliferative cells have the ability to form floating colonies, so-called spheres, when grown in nonadherent conditions. Furthermore, the spheres can be propagated for multiple generations, which is an indication that they are generated from self-renewing stem cells, very similar to neurospheres that grow from neural stem cells (McKay 1997). Regardless of the remarkable plasticity of neural stem cells, inner earderived stem cells and their derivatives are probably most suitable for use in transplantation experiments to replace lost hair cells because the inner ear-derived spheres contain cells that readily differentiate into hair cell-like cells in vitro and in vivo (Li et al. 2004a). One of the major obstacles that stem cell transplantation technology must overcome to repair damaged mammalian cochleae is the generation of hair cells in the organ of Corti in vivo, and despite some observations of neural stem cell survival and occasional expression of hair cell markers (Fujino et al. 2004; Parker and Cotanche 2004; Tateya et al. 2003), no robust regimen has been developed hitherto that allows the study of 
functional replacement of lost hair cells with stem cells. Nevertheless, the development of new surgical procedures and the exploration of stem cell types other than neural stem cells will hopefully result in more studies toward determining the potential of cochlear stem cell therapy.

A second major obstacle is the lack of a source of appropriate human stem cells for transplantation experiments into the cochlea and potential future therapy. Human embryonic stem cells could be such a source but are subjected to ethical criticism. Stem cells isolated from the human inner ear, on the other hand, could serve as a source without ethical constraints. Current research on this topic focuses on human inner ear tissue that is removed during infrequent inner ear surgery (Rask-Andersen et al. 2005). This strategy is hampered by the fact that surgically removed tissue is taken from patients with inner ear disease.

Toward exploring new sources for mammalian inner ear stem cells, we performed a pilot study in newborn mice to assess viability and features of cochlear and vestibular stem cells as a function of the postmortem interval, which could determine reasonable time limits for harvesting human inner ear tissues from autopsy cases in the future. Our results show that stem cells in the inner ear of newborn mice are detectable, alive, and functional up to $240 \mathrm{~h}$ after death. We therefore hypothesize that it should be possible to harvest human inner ear stem cells from autopsy cases, at least within the first few days postmortem. Although the therapeutic value of inner ear stem cells has not been determined, we propose that new sources, particularly for human inner ear stem cells, will advance the development of novel treatment strategies of inner ear diseases.

\section{MATERIAL AND METHODS}

\section{Animals}

Newborn (P1/P2) or 3-week-old (P21) Math-1/nuclear green fluorescence protein (nGFP) mice (Lumpkin et al. 2003) were euthanatized by $\mathrm{CO}_{2}$ overdose. To ensure death, we exposed the animals to $100 \% \mathrm{CO}_{2}$ for $2 \mathrm{~h}$, which is more than twice the maximum time to death reported for newborn neonatal mice with this procedure (Pritchett et al. 2005). Death was confirmed by absence of respiration, cessation of heartbeat, lack of reflexes, and loss of bladder control. Inner ear tissues were either dissected immediately postmortem or stored for $6 \mathrm{~h}$ at room temperature followed by storage at $4^{\circ} \mathrm{C}$ for later dissection. This storage scenario was chosen to simulate the conditions found in human autopsy cases. The study was performed with newborn and 3-week-old mice because we wanted to be able to investigate the postmortem features of all inner ear tissues, including the cochlea. Cochlear stem cells exist but are scarce in adult animals (Oshima et al. 2007). Postmortem intervals are defined as immediately after the $\mathrm{CO}_{2}$ exposure and decapitation $=0 \mathrm{~h}$, after $6 \mathrm{~h}$ room temperature $=6 \mathrm{~h}$, and storage at $4^{\circ} \mathrm{C}$ for $1,2,3,4,5,10$, and 15 days. Animal studies were conducted under an approved institutional protocol according to National Institutes of Health guidelines.

\section{Isolation of inner ear stem cells and sphere formation}

The utricular maculae, organs of Corti, and spiral ganglia were carefully dissected and transferred into a small drop of sterile, chilled phosphate-buffered saline (PBS) and cleaned from surrounding tissues (Fig. 1). All inner ear organs were then subjected to a 5-10-min treatment with $0.125 \%$ trypsin in $\mathrm{PBS}$ at $37^{\circ} \mathrm{C}$ in a total volume of $100 \mu \mathrm{l}$ for each organ. The enzymatic reaction was blocked by adding $100 \mu \mathrm{l}$ of $0.5 \%$ soybean trypsin inhibitor (Worthington, Lakewood, NJ, USA) and $2 \mathrm{mg} / \mathrm{ml}$ DNAse I (Worthington) in Dulbecco's modified Eagle's medium (DMEM)/highglucose and F12 media (mixed 1:1, Invitrogen, Carlsbad, CA, USA). The cells were carefully triturated $20-50 \times$ with plastic pipette tips (epTIPS Filter 20-300 $\mu \mathrm{l}$, Eppendorf, Hamburg, Germany). Cell separation was insured by microscopic inspection. Ninety-five percent of the cell suspension was used for sphere formation in a suspension cell culture, and $5 \%$ of the suspension was used to determine cell numbers and the ratio of living vs dead cells (see below).

Sphere formation was initiated by adding $2 \mathrm{ml}$ DMEM/high-glucose and F12 media (mixed 1:1) supplemented with N2 and B27 supplements (Invitrogen), EGF $(20 \mathrm{ng} / \mathrm{ml})$, basic fibroblast growth factor (bFGF) $(10 \mathrm{ng} / \mathrm{ml})$, insulin-like growth factor-1 (IGF-1) $(50 \mathrm{ng} /$ $\mathrm{ml}$ ), and heparan sulfate $(50 \mathrm{ng} / \mathrm{ml}$ ) (all growth factors were obtained from R\&D Systems, Minneapolis, MN, USA, and Sigma, St. Louis, MO, USA). The diluted suspension was then passed through a $70-\mu \mathrm{m}$ cell strainer (BD Labware, San Jose, CA, USA) directly into plastic Petri dishes (Greiner, Monroe, NC, USA), resulting in a single cell solution virtually devoid of large cell aggregates. Routinely, we plated the total yield of single cells that we obtained from the two inner ear organs of a single mouse into one Petri dish (Greiner Bio-One, suspension culture plate). The total numbers of primary spheres were assessed after 7 days in culture in a humidified incubator at $37^{\circ} \mathrm{C}$ and $5 \% \mathrm{CO}_{2}$.

Five percent $(10 \mu \mathrm{l})$ of the undiluted cell suspension was used for determination of cell numbers immediately after dissection and trituration. The numbers of living and dead cells were assessed using 


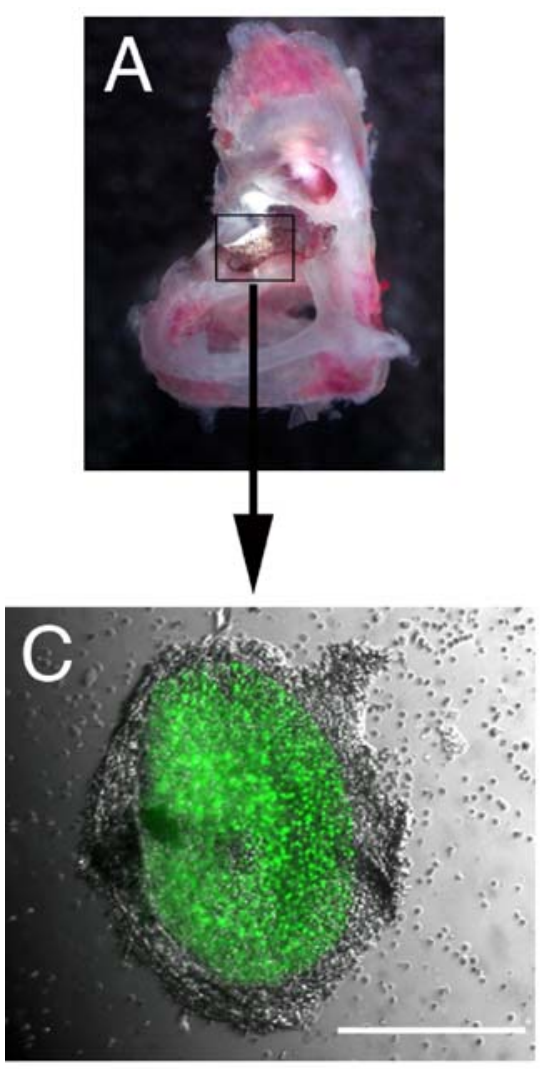

Utricle

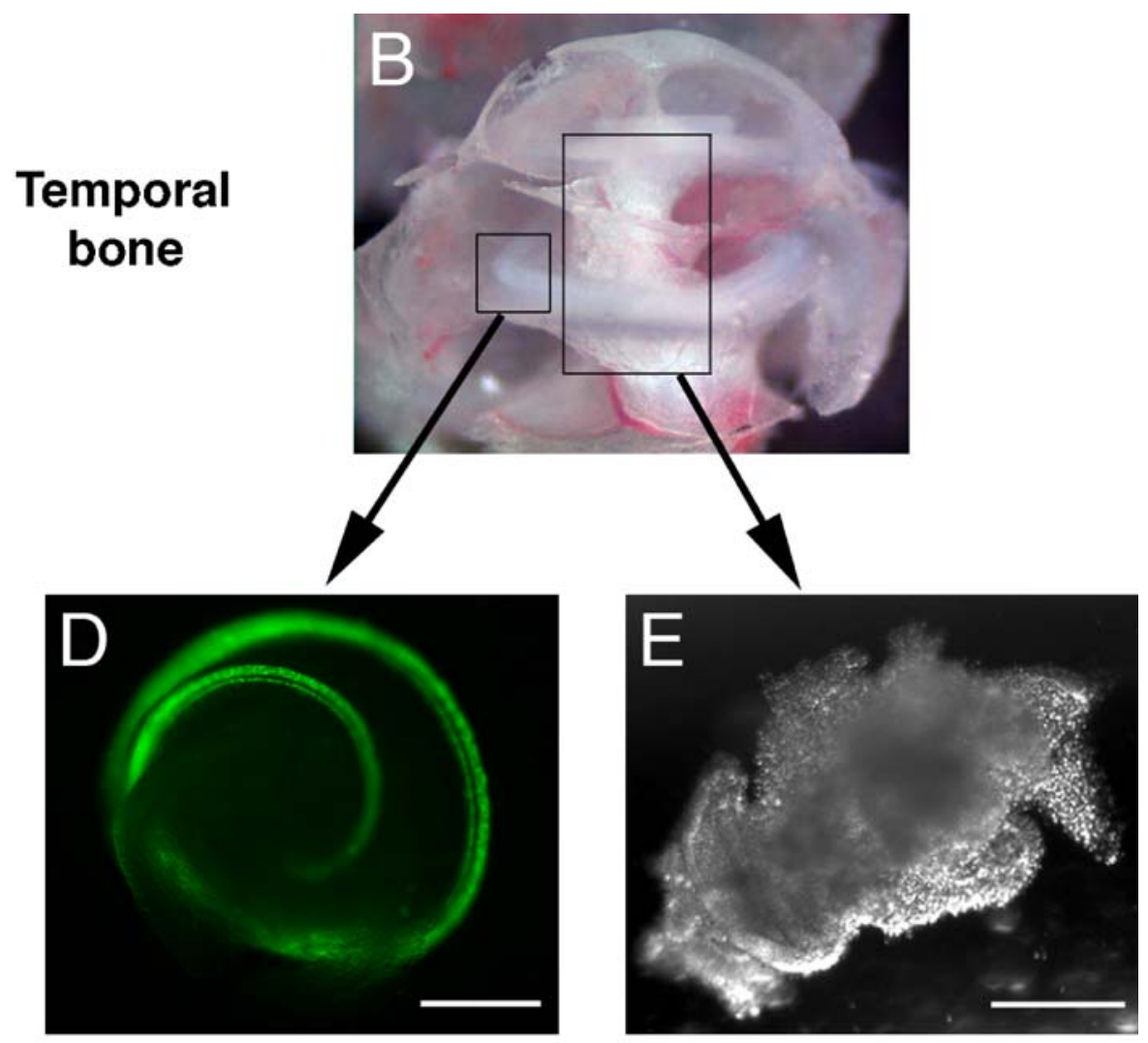

Organ of Corti

Spiral ganglion

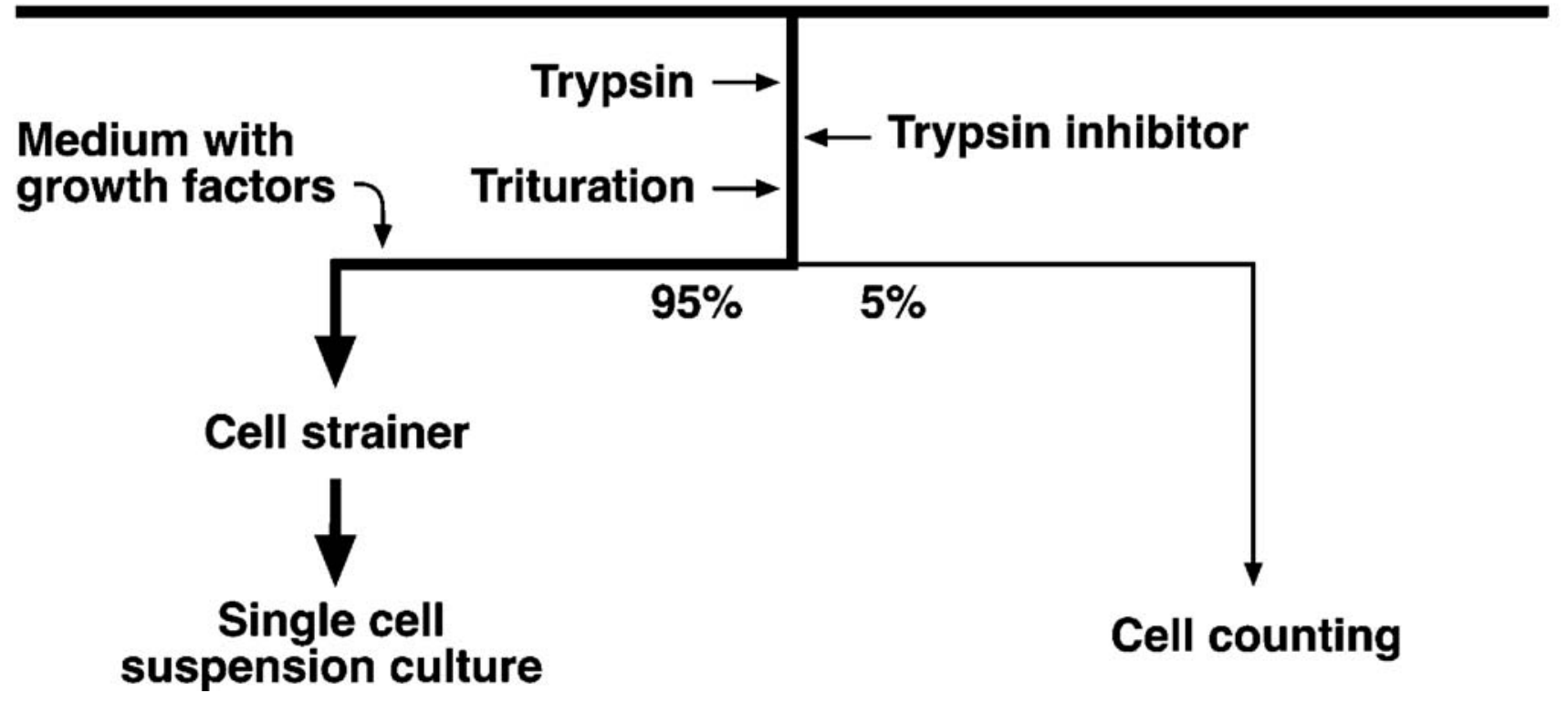

FIG. 1. Tissue isolation and cell handling procedure. A Temporal bone with utricle (box). B Cochlea. Positions of a sector of the organ of Corti (small box) and the spiral ganglion (large box) are indicated. C-E Dissected utricular macula, organ of Corti, and spiral ganglion. nGFP-positive hair cells are visualized by green fluorescence. Flow chart, generation of single cell suspension cultures. Images show specimen dissected at $0 \mathrm{~h}$ postmortem from 3-week-old (A, B) and newborn (C-E) mice. Scale bars $=300 \mu \mathrm{m}$. 
a hemacytometer (Neubauer improved) after adding $10 \mu \mathrm{l}$ of a $0.4 \%$ solution of trypan blue (Invitrogen) to the single cell solution. Exclusion of the dye from living cells allowed us to determine the absolute and relative number of dead vs living cells.

Due to the frailness of the tissues at later postmortem stages, we had to modify our previously published dissection protocol (Oshima et al. 2007). Oshima et al. dissected the inner ear in a dish with Hanks' solution and transferred the cleaned inner ear organs into a drop of PBS before adding trypsin. In the present protocol, we dissected and cleaned the inner ear organs directly in a small drop of PBS before adding trypsin to the same drop. Using this method, we made sure that we did not lose frail inner ear tissues during the cleaning step. We believe that this method may lead to an increase in the number of cells from surrounding tissue, which remain swimming in the drop. Therefore, cell numbers per specimen cannot be directly compared to the numbers published by Oshima et al. (2007). Additionally, we also would like to note that we present sphere numbers as spheres per $10^{4}$ living cells as compared to earlier papers from our laboratory, which reported sphere numbers per $10^{4}$ total cells ( $\mathrm{Li}$ et al. 2003a; Oshima et al. 2007).

\section{Stem cell propagation}

Spheres derived from spiral ganglia were propagated by dispersing 30-50 spheres with a 5-min Accumax (Innovative Cell Technologies, San Diego, CA, USA) treatment at $37^{\circ} \mathrm{C}$ followed by careful trituration using a fire-polished glass pipette. In contrast, utricular and organ of Corti-derived spheres were mechanically dissociated after treatment with Accumax for $9 \mathrm{~min}$ at $37^{\circ} \mathrm{C}$. Accumax activity was blocked by diluting the cell suspension with media. The cell suspensions were replated into DMEM/high-glucose and F12 media (mixed 1:1) supplemented with N2 and B27 supplements (Invitrogen), EGF (20 ng/ml), bFGF $(10 \mathrm{ng} / \mathrm{ml})$, IGF-1 $(50 \mathrm{ng} / \mathrm{ml})$, and heparan sulfate $(50 \mathrm{ng} / \mathrm{ml}$ ) (growth factors and heparan sulfate were obtained from Sigma) in Petri dishes and propagation was repeated at 5-day intervals. Fresh growth factors were added daily from stock solutions. For long-term propagation, we counted the number of spheres for each generation, related the number to the original sphere number, and replated half of the spheres for producing the next generation.

\section{Cell differentiation}

Second- or third-generation spheres were plated onto fibronectin-coated Lab-Tek ${ }^{\circledR} I I$ slides (Chamber Slide ${ }^{\mathrm{TM}}$ system, Nalge Nunc International, Rochester,
NY, USA) in DMEM/high glucose and F12 medium (mixed 1:1) supplemented with N2 and B27 supplements. Eighty percent of the medium was replaced twice a week. Differentiated cells were analyzed after 14 days by immunocytochemistry.

\section{Immunocytochemistry}

The attached cells were fixed for 5 min with $4 \%$ paraformaldehyde in PBS and washed with PBS, and nonspecific binding sites were blocked for $1 \mathrm{~h}$ in $0.1 \%$ Triton-100, $1 \%$ bovine serum albumin (BSA) (wt/vol), and $5 \%$ (wt/vol) heat-inactivated goat serum in PBS (PBT1). The slides were incubated overnight at $4^{\circ} \mathrm{C}$ in PBT1 with diluted antibodies: 1:1,000 for polyclonal guinea pig antibody to murine myosin activated factor VII (VIIA) (Oshima et al. 2007), 1:2,000 for polyclonal rabbit antibody to parvalbumin 3 (Heller et al. 2002), 1:3,000 for polyclonal antibody to espin ( $\mathrm{Li}$ et al. 2004b), 1:500 for polyclonal antibody to glial fibrillary acidic protein (GFAP; Dako, Glostrup, Denmark), and 1:500 for monoclonal mouse antibody to neuron-specific $\beta$-III tubulin (TuJ; Covance, Princeton, NJ, USA). Cy5-, tetramethyl rhodamine iso-thiocyanate-, and flourescein isothiocyanate-conjugated secondary antibodies (Jackson Immuno Research, West Grove, PA, USA) were diluted $1: 400$ in $0.1 \%$ Triton- 100 and $0.1 \%$ BSA (wt/vol) in PBS and used to detect primary antibodies. 4',6-Diamidino-2phenylindole dihydrochloride (DAPI; Invitrogen) was used to visualize nuclei. The slides were analyzed using an epifluorescence microscope equipped with digital camera and image acquisition software (Zeiss Axio Imager with AxioCam HR and AxioVision 4.0 running on a Fujitsu-Siemens PC).

\section{Immunohistochemistry}

Temporal bones were dissected and the otic capsule was opened by drilling a small opening at the helicotrema and by removing the stapes footplate. After fixation overnight with $4 \%$ paraformaldehyde in PBS, the specimens were washed with PBS, soaked in $15 \%$ sucrose (wt/vol) in PBS for $6 \mathrm{~h}$, and transferred to a $30 \%$ sucrose (wt/vol) solution in which they remained overnight at $4^{\circ} \mathrm{C}$. The cryoprotected specimens were then embedded in OCT compound (Tissue-Tek, Sakura Finetek, Torrance, CA, USA), frozen at $-20^{\circ} \mathrm{C}$, and cryosectioned with a thickness of 12-16 $\mu \mathrm{m}$ (CM3050S cryostat, Leica, Wetzlar, Germany). The slides were incubated overnight with diluted primary antibodies that were visualized with fluorescent dyeconjugated secondary antibodies as described in the preceding section. Rhodamine-conjugated phalloidin (Invitrogen) was used to stain filamentous actin and DAPI was used to visualize nuclei. The specimens were 
examined and documented with an epifluorescence microscope as described above.

\section{RESULTS}

Morphology and cell survival in postmortem inner ear tissues

We studied the morphology of inner ear tissues directly after death and after postmortem intervals of 5 days (data not shown) and 10 days (Fig. 2A-E) to better understand the progression of tissue degeneration and the possibility of cell survival. As expected, we found a progressive loss of organization and a loosening of tissue density with increasing postmortem intervals. Despite these apparent degenerative changes, the overall architecture of inner ear organs and the morphology of many individual cells remained surprisingly well preserved after 10 days postmortem. Within the vestibular and auditory sensory epithelia, we found cells of defined shape with filamentous actin-containing hair bundles that were immunopositive for the hair bundle marker espin (Roblin et al. 2003; Zheng et al. 2000). These hair cells displayed green fluorescence, indicative of the nGFP reporter that is expressed in the nuclei of the transgenic Math1/nGFP mouse line (Lumpkin et al. 2003) used for our study. Nevertheless, at 10 days postmortem, the distribution of nGFP was typically no longer mainly nuclear (Fig. 2B, D) and the hair bundles often appeared detached from the remainder of the cell. Overall, there was substantial loss of hair cells both in the utricle and in the organ of Corti. In the spiral ganglion, we observed massive loss of neural cell bodies, accompanied by a disappearance of the neural marker $\beta$-III tubulin (Fig. 2E).

To quantify cell survival in the inner ear as a function of the postmortem interval, we dissected utricular maculae, organs of Corti, and spiral ganglia of Math1/nGFP mice directly after death and at various time points postmortem. We ensured complete inclusion of the individual organs, transferred them into a single drop of sterile PBS, and carefully removed excessive tissue. This procedure, a modification of previous protocols ( $\mathrm{Li}$ et al. 2003a; Oshima et al. 2007), was necessary because the tissue became very frail, particularly with increasing postmortem intervals. After the cells were dissociated, the total cell number per specimen was determined (Fig. 3). Surprisingly, the total number of cells in all tissues examined did not significantly decrease during the first 10 days postmortem. At 15 days postmortem, the total cell number in the organ of Corti and spiral ganglion was significantly lower than the numbers directly after the death of the animal (Fig. 3). The ratio of living vs dead cells in the total cell population declined gradually from
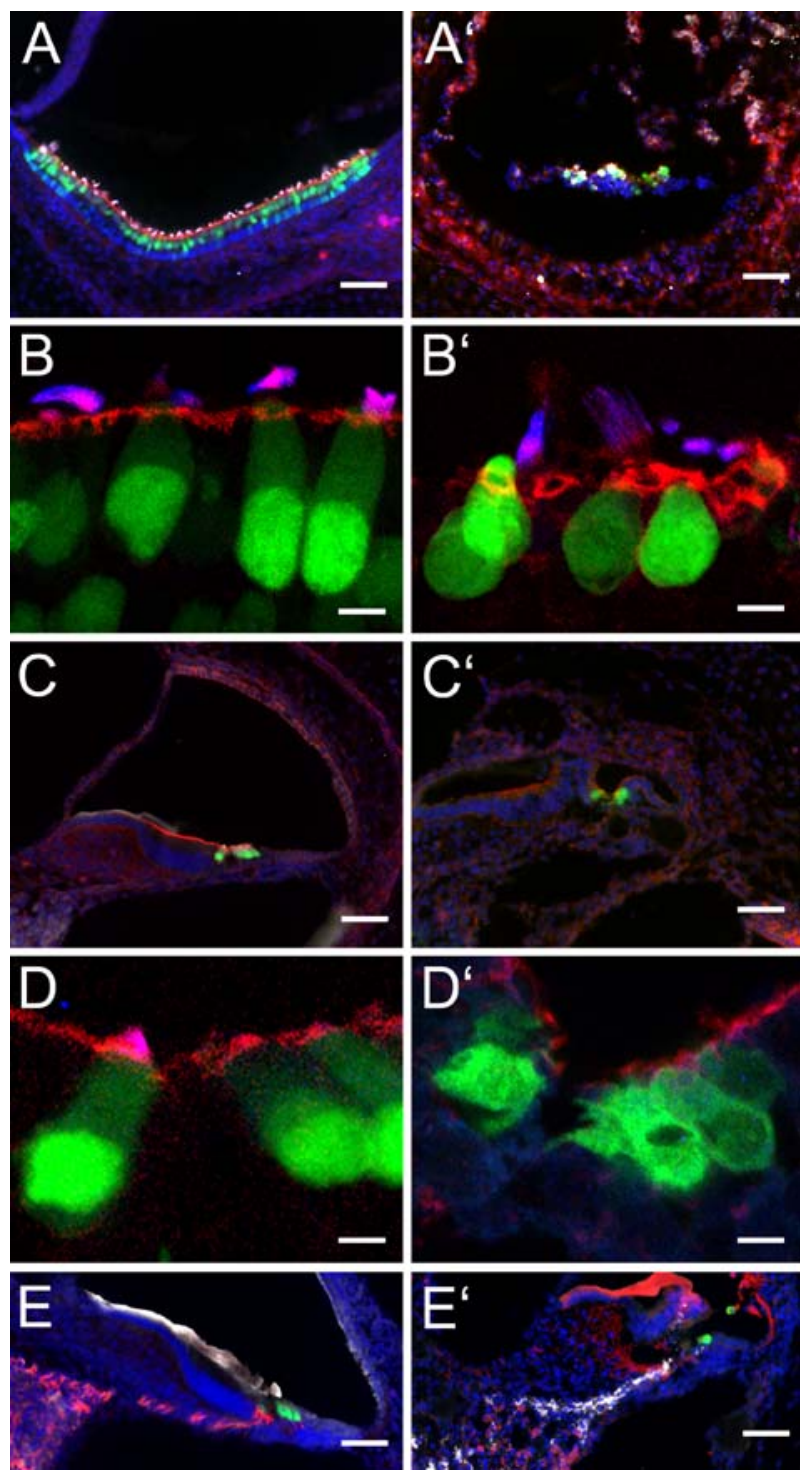

FIG. 2. Cross-sections through the inner ear of newborn Math $1 /$ nGFP mice at $0 \mathrm{~h}(\mathbf{A}-\mathbf{E})$ and 10 days postmortem $\left(A^{\prime}-E^{\prime}\right)$. A, $A^{\prime}$ Shown is the utricle with nGFP-positive hair cells (green). Filamentous actin is visualized in red, and the hair bundle protein espin, detected with Cy5-conjugated secondary antibody, is depicted in white. Nuclei are visualized with DAPI (blue). B, $B^{\prime}$ Higher magnification of utricular hair cells reveals loss of nuclear confinement of nGFP (green) at 10 days postmortem. Hair bundles are visualized with antibody to espin (blue) and by labeling with rhodamine-conjugated phalloidin (red) to detect filamentous actin. C, $C^{\prime}$ Organ of Corti sections and D, $D^{\prime}$ higher magnification images of cochlear hair cells. $\mathbf{E}, E^{\prime}$ In the spiral ganglion, we observed massive loss of $\beta$-III tubulin (TuJ)-immunopositive neural cell bodies and their associated neurites (depicted in red). GFAP immunoreactivity (shown in white) was present at $0 \mathrm{~h}$, although not very pronounced, but appeared stronger at 10 days postmortem than at $0 \mathrm{~h}$. Note that the labeling pattern of antibodies to TuJ and to GFAP appears to be altered at 10 days postmortem, possibly due to extensive autolytic processes that probably reveal novel unspecific antigens in the tissue. The apparent positive immunoreactivity of the tectorial membrane is unspecific because it is also detectable in negative controls where the primary antibodies were omitted. nGFPpositive hair cells are shown in green and nuclei are visualized with DAPI (shown in blue). Scale bars $=50 \mu \mathrm{m}$ in $\mathbf{A}, A^{\prime}, \mathbf{C}, C^{\prime}, \mathbf{E}$, and $E^{\prime}$ and $10 \mu \mathrm{m}$ in $\mathbf{B}, B^{\prime}, \mathbf{D}$, and $D^{\prime}$. 

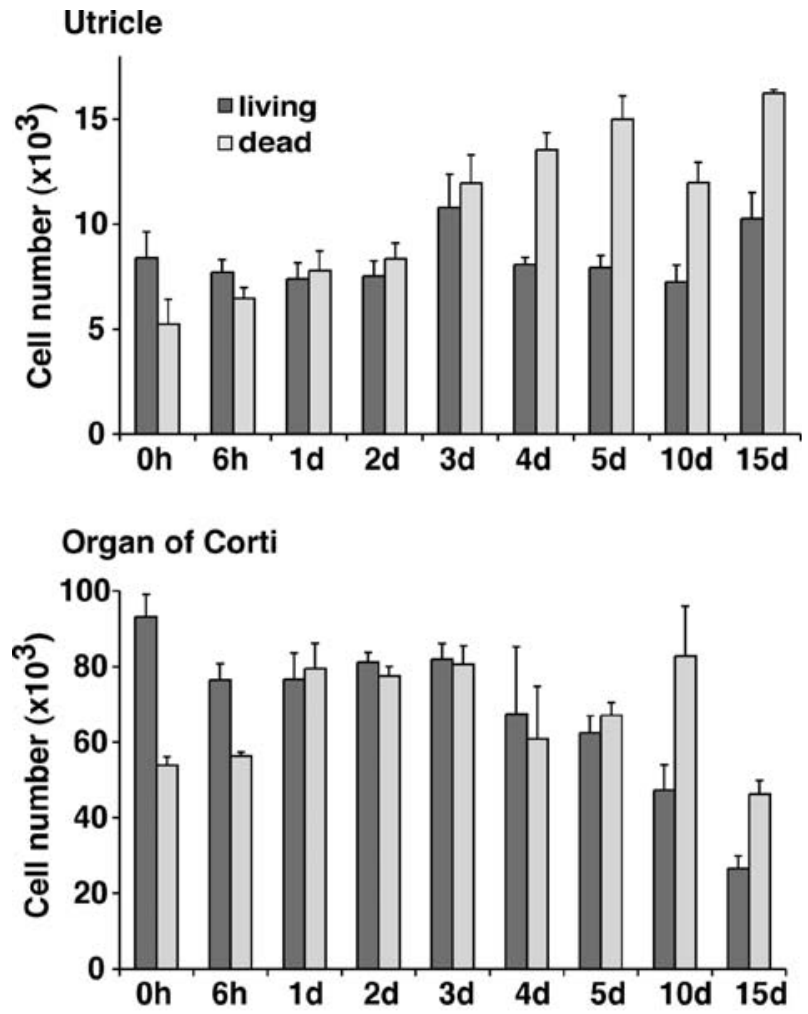

\section{Spiral ganglion}

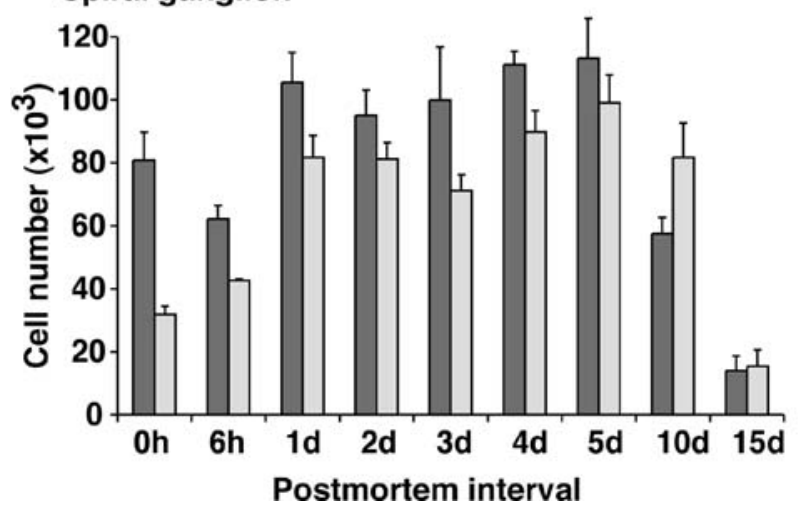

FIG. 3. Total number of cells per individual utricle, organ of Corti, and spiral ganglion after generation of a single cell suspension and removal of aggregates. Shown are mean values \pm SEM, $n=3$.

$0.62-0.68$ at $0 \mathrm{~h}$ postmortem to $0.28-0.41$ at 15 days postmortem.

Sphere formation as a function of the postmortem interval

The vigorous incidence of surviving cells in the inner ear sensory epithelia and the spiral ganglion after protracted postmortem intervals encouraged us to explore whether cells with the ability to form floating spheres could remain viable as well. Indeed, we found that cell suspensions prepared from the individual inner ear organs harvested up to 10 days postmortem robustly gave rise to free-floating spheres in serum-free culture (Figs. 4A and 5). Spheres from the utricle and the organ of Corti were either solid in appearance with a smooth surface or solid with a rough surface, whereas spheres derived from the spiral ganglia appeared exclusively solid and roughsurfaced, similar to clusters of grapes. The overall appearance of spheres did not noticeably change when we compared spheres that formed from cells isolated immediately, 5 days, or 10 days postmortem. In cultures derived from cells isolated from utricles and organs of Corti, we found a substantial number of hollow spheres (Fig. 5). These hollow spheres, however, showed no ability to self-renew or to differentiate into hair cell or neuron marker-positive cells (see below), and all data presented in the remainder of the paper refer to solid spheres.

Generally, the utricle yielded lower numbers of sphere-forming cells per $10^{4}$ cells than the organ of Corti and the spiral ganglion. The number of spheres per $10^{4}$ living cells from the utricle was $91.6 \pm 14.9$ (mean \pm SEM; $n=3$ ) directly after death and did not change significantly during the first 4 days postmortem. At 5 and 10 days postmortem, the utricle had lost about $60 \%$ of its ability to give rise to spheres and
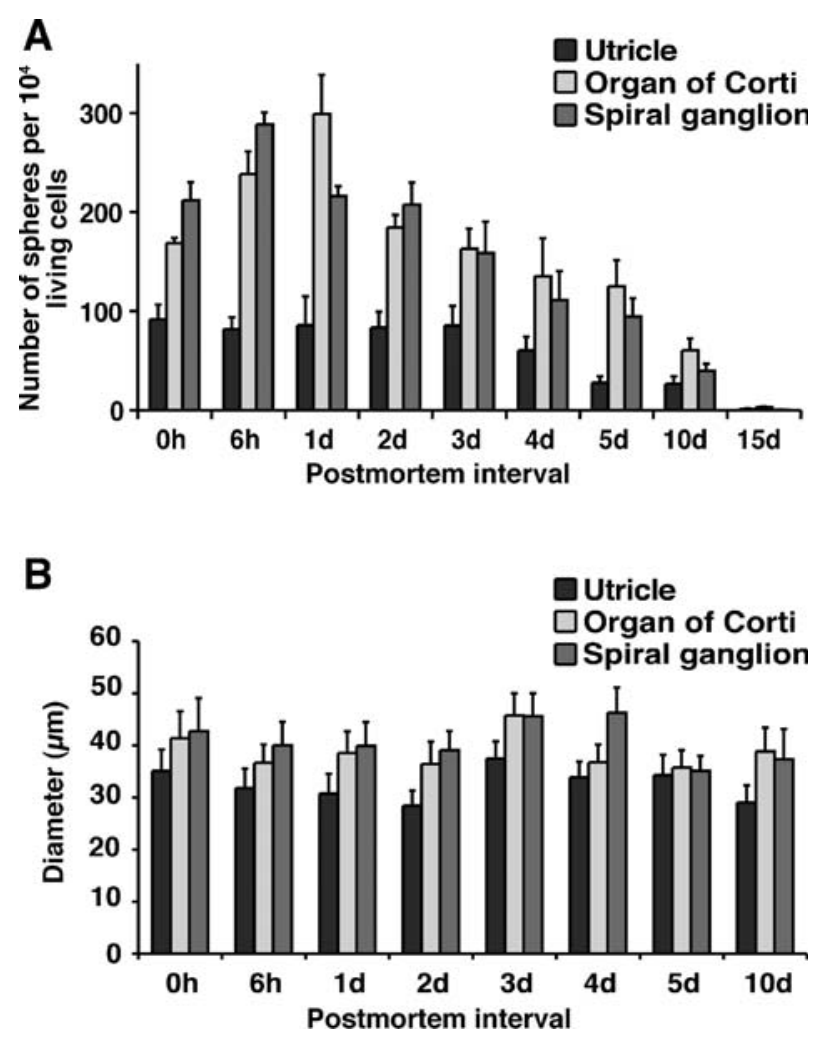

FIG. 4. Primary spheres from postmortem tissues. A Shown are the number of solid primary spheres per $10^{4}$ living cells that formed after 7 days in nonadherent serum-free cultures. B Diameters of solid primary spheres at different postmortem time intervals. Shown are mean values \pm SEM, $n=3$. 


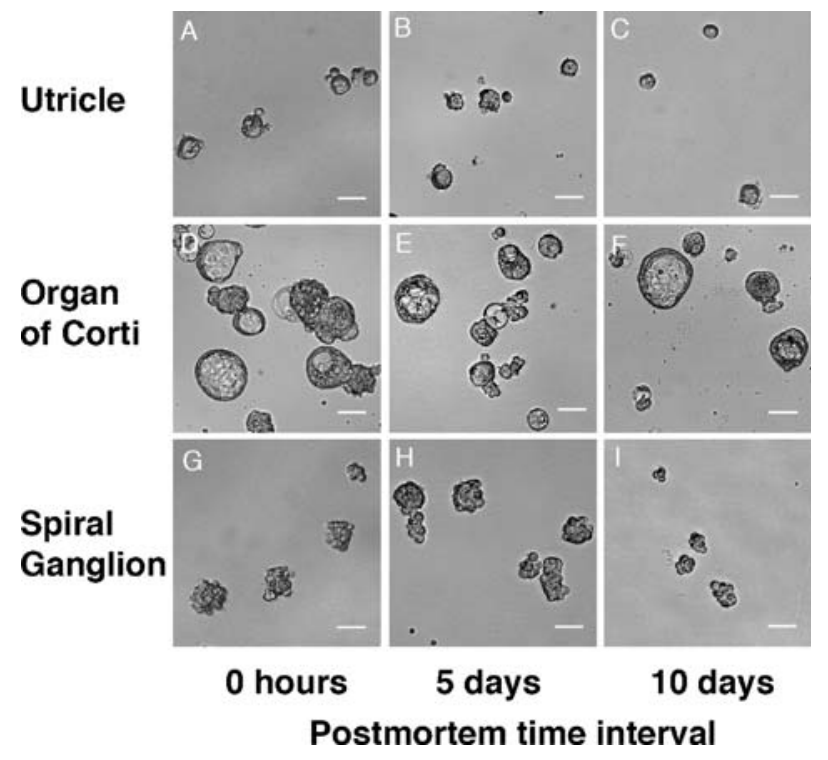

FIG. 5. Shapes of spheres derived from utricle (A-C), organ of Corti (D-F), and spiral ganglion $(\mathbf{G}-\mathbf{I})$ directly postmortem $(0 \mathrm{~h})$ and after 5 and 10 days. Hollow spheres that did not self-renew were found in preparations from the utricle and organ of Corti and are shown in $\mathbf{D}$, $\mathbf{E}$, and F. Hollow spheres were not included in the sphere size measurements shown in Figure 4 and were not used for further experiments. Scale bars $=40 \mu \mathrm{m}$.

only one single sphere in three independent experiments was generated from 15-day-postmortem utricular cells. In both the organ of Corti and the spiral ganglion, we noted a higher incidence of sphereforming cells than in the utricle [168.5 \pm 5.4 and $211.8 \pm$ 18.2 (mean \pm SEM; $n=3$ ) spheres per $10^{4}$ living cells, respectively] and increasing numbers of sphereforming cells during the first $24 \mathrm{~h}$ after death (Fig. 4A). Similar to the utricle, the organ of Corti and the spiral ganglion displayed reduced ability to generate spheres after 5 and 10 days postmortem. Only occasionally did we observe formation of spheres with cells isolated at day 15 postmortem: in two of three cases, we found a single sphere after plating $10^{4}$ cells of a cell suspension prepared from day-15postmortem organs of Corti. Interestingly, we found a tendency for higher sphere numbers in organ of Corti and spiral ganglion tissues harvested at $6 \mathrm{~h}$ and 1 day postmortem when compared with those harvested immediately postmortem. We hypothesize that postmortem-related changes in the dying tissue, such as hypoxia, acidosis, and lack of nutrients, may stimulate or positively select stem cells over other tissue cells.

We have previously shown that utricle-derived spheres can form from single cells (Li et al. 2003a). Using flow cytometry, we verified that organ of Cortiderived solid spheres also form from single cells with an incidence of 30 spheres per $10^{4}$ cells. This number is smaller than the number we obtained when plating the cells at low density, which is an indication that inner ear stem cells are sensitive to the flow cytometry procedure or that some spheres in our experiments may start out as small cell aggregates.

The apparent postmortem changes in the numbers of spheres per $10^{4}$ cells led us to investigate whether spheres derived at different postmortem periods differed in their growth potential. A simple measure of a sphere's growth potential is its diameter at a fixed time after plating. We found that the diameters of solid spheres of all three inner ear organs did not significantly change when compared at different postmortem intervals (Fig. 4B). The diameters of utricle-derived spheres ranged from $28.4 \pm 2.9$ to $37.5 \pm 3.3 \mu \mathrm{m}$ (mean $\pm \mathrm{SEM} ; n=3$ ), organ of Cortiderived spheres ranged from $35.7 \pm 3.4 \mu \mathrm{m}$ to $45.7 \pm$ $4.2 \mu \mathrm{m}$ (mean \pm SEM; $n=3$ ), and spiral ganglion-derived spheres ranged from $35.1 \pm 2.9$ to $46.2 \pm 4.9 \mu \mathrm{m}$ (mean \pm SEM; $n=3$ ).

The self-renewal capacity of inner ear stem cells does not change postmortem

Self-renewal is the defining feature of stem cells, and we have previously shown that inner ear-derived stem cells can be propagated by mechanical dissociation of primary spheres, which resulted in the formation of secondary spheres that could be propagated again for many generations ( $\mathrm{Li}$ et al. 2003a; Oshima et al. 2007). The robust generation of spheres after protracted postmortem intervals raised the question whether spheres derived from postmortem tissues could also be expanded by mechanical dissociation, which would demonstrate that these spheres were formed from self-renewing stem cells. We therefore propagated spheres generated from the utricle, organ of Corti, and spiral ganglion for at least three generations and determined the ratio by which we were able to expand the spheres from one generation to the next (Fig. 6). Only spheres of solid appearance could be propagated with this method. Mechanical dissociation of hollow spheres did not lead to a new generation of spheres-the cells died and the propagation ratio for these spheres was zero. Solid spheres from all three tissues could be propagated with a ratio between 1.2 and 2 next-generation spheres per individual sphere. We found no statistical difference in the propagation capacity when we compared spheres from all three tissues investigated directly after death with spheres derived from 5- and 10-daypostmortem tissues. These results imply that solid spheres contain renewing cells and that the capacity for self-renewal of these stem cells does not change during the 10-day postmortem interval that we investigated. 


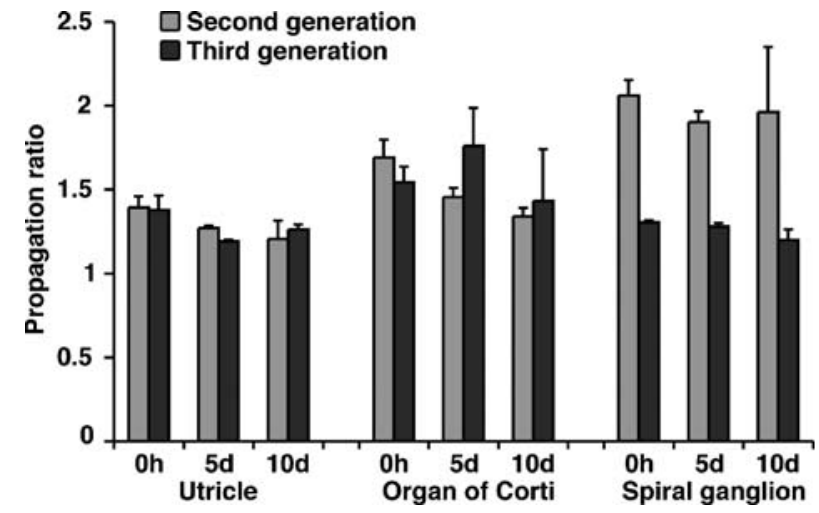

FIG. 6. Propagation and expansion of spheres from utricle, organ of Corti, and spiral ganglion into the second and third generation. Shown is the increase of second- and third-generation sphere numbers from a single sphere. Shown are mean values \pm SEM, $n=3 ; 30-50$ spheres were used for each individual experiment.

Spheres derived from postmortem tissues give rise to mature inner ear cell types but lose the ability to generate differentiated cells after protracted postmortem intervals

Sphere-forming stem cells isolated from the adult mouse utricle are capable of differentiating into several different cell types, including hair cell-like cells and neurons ( $\mathrm{Li}$ et al. 2003a). This multipotency is also characteristic of organ of Corti- and spiral ganglionderived stem cells because organ of Corti-derived stem cells can be differentiated into hair-like cells and supporting cells and spiral ganglion-derived stem cells into neurons and cells that express glial markers (Oshima et al. 2007). We were interested in exploring whether stem cells isolated after protracted postmortem intervals remain multipotent and whether their potency to generate hair cell marker-expressing cells and neurons remains stable when compared with stem cells isolated directly after death of the animal. Robust maintenance of multipotency after prolonged postmortem intervals would be a desirable feature, particularly for planned future tests with human inner ear tissue.

For this experiment, we propagated spheres derived from the utricle, the organ of Corti, and the spiral ganglion until the second or third generation. We then allowed the spheres to attach to fibronectincoated cell culture dishes and we deprived the attached cells of mitogenic factors. After culturing the cells for 2 weeks in these conditions, which were previously utilized to induce hair cell and neuronal differentiation ( $\mathrm{Li}$ et al. 2003a; Li et al. 2003b; Oshima et al. 2007), we analyzed the cells for the expression of specific markers. As expected, attached spheres from the 0-h-postmortem utricle and organ of Corti robustly gave rise to hair-cell-like cells, defined by coexpression of nGFP and myosin VIIA (Fig. 7A, E), and to neurons (data not shown). Spheres from the spiral ganglion did not give rise to hair cell markerpositive cells (data not shown) but generated neurons and glial fibrillary acidic protein (GFAP)-positive cells (Fig. 7I). We did not find major differences in the numbers of differentiated hair-cell-like cells derived from 5-day-postmortem utricle and organs of Corti, when compared with those from $0 \mathrm{~h}$ postmortem (Fig. 7A-H); the number of neurons and GFAP-positive cells generated from 5-day-postmortem spiral gangliaderived spheres was reduced by $\sim 50 \%$ (Fig. $7 \mathrm{I}-\mathrm{L}$ ). Ten days postmortem, we found that utricle-derived spheres failed to attach to the fibronectin substrate; therefore, we were not able to determine the differentiation potential of these spheres. Organ of Corti-derived spheres, however, were still able to produce hair cell marker-expressing cells at 10 days postmortem (Fig. 7G, H). Spiral ganglion spherederived cell populations lost their ability to generate neurons and GFAP-positive cells (Fig. 7K, L). Overall, these results show that inner ear sensory epitheliaderived spheres retain the ability to generate haircell-like cells and that spiral ganglion-derived spheres retain the ability to generate neuronal cell types for at least 5 days postmortem. Similar to our previous observations (Li et al. 2003a; Oshima et al. 2007), we found that the multipotency of inner ear stem cells in vitro is restricted. Utricle- and organ of Corti-derived stem cells seem to be able to give rise to hair-cell-like cells and, to a lesser extent, to neurons (data not shown). Spiral ganglion-derived stem cells do not give rise to hair-cell-like cells in vitro but robustly differentiate into neurons and glial cell types.

\section{Spheres from postmortem tissues of older mice}

A prospective goal of our research is to explore the merit of potential human inner ear stem cells. Because our study was conducted with neonatal mice, one could argue that our results are not directly comparable with the human situation where postmortem tissue will likely be obtained from adults. Conversely, the use of neonatal mice allowed us to investigate the postmortem survival of cochlear stem cells, which are rarely found in adult mice (Oshima et al. 2007).

To show that sphere-forming stem cells are also present in the postmortem inner ear of older mice, we chose three-week-old (P21) mice because, at this age, sphere-forming stem cells can be isolated from vestibular and cochlear parts of the inner ear (Oshima et al. 2007). We found that the P21 utricle harbors $13.5 \pm 1.2$ (mean $\pm \mathrm{SEM} ; n=3$ ) sphere-forming cells per $10^{4}$ living cells $0 \mathrm{~h}$ postmortem, $5.9 \pm 1.3$ sphere- 


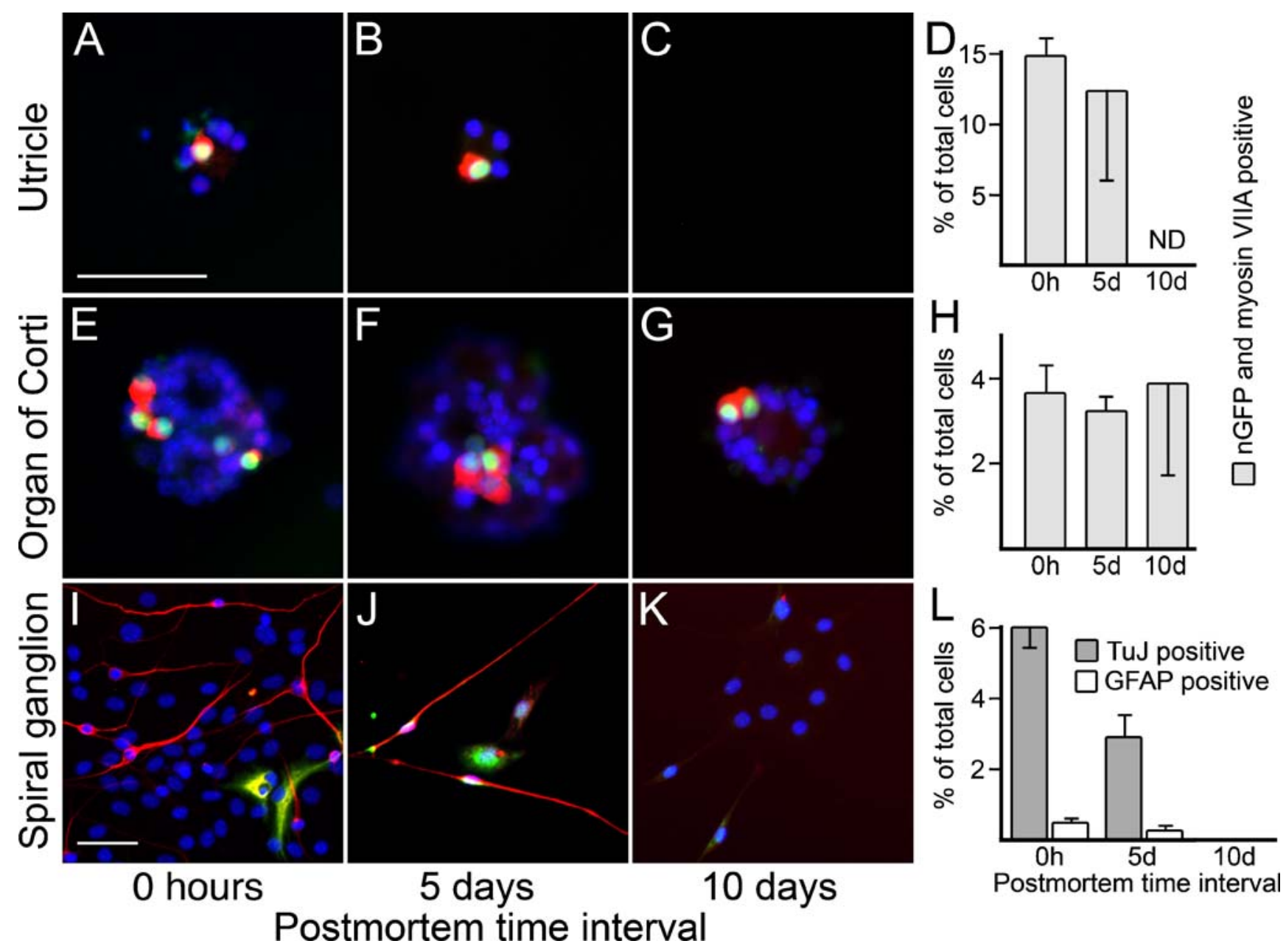

FIG. 7. Differentiation of mature cell types from spheres derived at different postmortem time intervals. (A-B) Hair-cell-like cells, defined by nGFP expression (green) and labeling with antibody to myosin VIIA (red) can be found in differentiated cells from utricle-derived spheres at $0 \mathrm{~h}$ and 5 days postmortem. C At 10 days postmortem, utricle-derived spheres failed to attach to the substrate. D Quantitative assessment of the number of nGFP and myosin VIIA double positive cells of the total cell number of differentiated utricle-derived spheres. E-G Occurrence of hair-cell-like cells in organ of Corti-derived spheres at $0 \mathrm{~h}$ and 5 and 10 days postmortem. $\mathbf{H}$ Quantitative assessment of the number of nGFP and myosin VIIA double positive cells of the total cell number of differentiated organ of Corti-derived spheres. I-K Neurons, defined by expression of $\beta$-III tubulin (TuJ, shown in red), and GFAP expressing cells (shown in green) can be found in differentiated cells from spiral ganglion-derived spheres at $0 \mathrm{~h}$ and 5 days postmortem, but not at 10 days postmortem. In some instances, we detected very weak expression of TuJ in GFAPpositive cells, which we interpret as a sign of immaturity of these newly generated cells. L Quantitative assessment of the number of TuJ- and GFAP-positive cells of the total cell number of differentiated spiral ganglion-derived spheres. Nuclei are visualized with DAPI (shown in blue); scale bars $=50 \mu \mathrm{m}$.

forming cells at 3 days postmortem, and $0.71 \pm 0.24$ sphere-forming cells at 5 days postmortem. In the organ of Corti, we found $2.1 \pm 0.3$ sphere-forming cells per $10^{4}$ living cells $0 \mathrm{~h}$ postmortem, $0.5 \pm 0.1$ sphere-forming cells at 3 days, and $0.2 \pm 0.1$ sphereforming cells at 5 days postmortem. Finally, in the spiral ganglion, we found $2.4 \pm 0.2$ sphere-forming cells per $10^{4}$ living cells $0 \mathrm{~h}$ postmortem, $1.1 \pm 0.1$ sphere-forming cells at 3 days postmortem, and $0.2 \pm 0.1$ sphere-forming cells at 5 days postmortem. Our 0-h-postmortem numbers are comparable with previous results, although the methods used for stem cell isolation were, to some extent, different (Oshima et al. 2007).

\section{DISCUSSION}

The main conclusion that we draw from our study is that inner ear stem cells are surprisingly robust and survive in reasonable numbers even after protracted postmortem time intervals. We were able to routinely isolate sphere-forming stem cells from vestibular and auditory sensory epithelia and from the spiral ganglion until 10 days postmortem. To demonstrate the self-renewal capacity and potency of inner ear stem cells, we propagated the spheres and tested secondand third-generation spheres for their ability to generate inner ear cell types defined by hair cell and neuronal marker expression. As expected ( $\mathrm{Li}$ et al. 
2003a; Oshima et al. 2007), sensory epitheliumderived spheres readily gave rise to hair cells and other cell types such as neurons (data not shown, but see Li et al. 2003a; Oshima et al. 2007), whereas spiral ganglion-derived neurospheres served as a potent source for neurons and glial cell types, but not for hair cells. This capacity of the different sphere types was determined by spontaneous differentiation in serum-free culture and we consider that the true potential of the different inner ear-derived spheres is higher when the cells are exposed to different environments. This hypothesis is supported by our previous finding that utricle-derived sphere-forming stem cells display pluripotency after grafting into a gastrulating embryo (Li et al. 2003a). Nevertheless, we also observed changes in the spheres derived from 10-day-postmortem tissues. Utricle-derived spheres lost their ability to attach to the fibronectin substrate that we used in our experiments and spiral ganglionderived spheres lost their ability for neural differentiation. These observations suggest that significant molecular changes happen after death that affect the potency of the sphere-forming utricle and spiral ganglion stem cells and their derivatives. Conversely, organ of Corti-derived sphere-forming stem cells did not show reduced potency to generate hair cell marker-positive cells, even after 10 days postmortem. We observed, however, a much higher interexperimental variability when quantifying hair cell markerpositive cells from 10-day-postmortem organ of Corti spheres. In addition, we also experienced a reduced ability of these spheres to attach to the fibronectin substrate (data not shown), which indicates that the progenitor and stem cells in these spheres likely undergo similar diminishing changes when compared with the 10-day-postmortem spheres from utricle and spiral ganglia, but with some delay.

It is remarkable that spheres generated from 5-daypostmortem tissues were virtually indistinguishable from spheres generated from tissues isolated directly after death. We did not detect any quantitative differences in the growth potential or in the ability to generate specific cell types. Postmortem changes in the sphere-forming cells appear to first reduce the cells' potency to differentiate into mature cell types before affecting the stem cells' growth potential. An explanation for this change in the cells' ability could be that their restricted potency between postmortem days 5 and 10 is a result of a fallback into a less committed mode, which would require additional signals to initiate specific cell differentiation.

\section{Stem cells after death}

Organ donation is a widely utilized and an obvious strategy to obtain organs for transplantation to treat heart, kidney, lung, and liver disease, among others. Some organs, such as the cornea and the middle ear ossicles, are removed postmortem and reused for allografting. It has been shown previously that splitthickness skin grafts can be allografted up to 3 weeks after death if stored at $4^{\circ} \mathrm{C}$ (Rosenquist et al. 1988), a fact that supports our results with surviving cells in the inner ear up to 15 days postmortem. More recently, stem and progenitor cells have been isolated from postmortem brain, spinal cord, and the retina (Laywell et al. 1999; Mayer et al. 2005; Palmer et al. 2001; Roisen et al. 2001). Generally, it appears that many stem cells survive for hours or even days after death, but no study thus far has systematically and quantitatively investigated the postmortem time course of the decrease in stem cell numbers and the specific potential of stem cells isolated at different postmortem time intervals. Moreover, we observed an increase in sphere numbers from organ of Corti and spiral ganglion after $6 \mathrm{~h}$ and 1 day postmortem (Fig. 4A), and we speculate that hypoxia, acidosis, or lack of nutrients and other postmortem-related factors may contribute to a stimulation or positive selection of stem cells over other cells in dying tissue. This stem cell activation may be the result of an effort to repair local tissue damage.

Our results show that it is possible to isolate sphere-forming stem cells not only from neonatal animals, but also from older mice (P21). This evidence spurs our confidence that the isolation of sphere-forming cells from the postmortem human inner ear will be a feasible endeavor in the near future. Nevertheless, we observed a reduction in the number of spheres generated from inner ear tissues of 3-week-old mice when we compared sphere formation directly following death with sphere formation at 3 and 5 days postmortem. From this observation, we conclude that the isolation of adult inner ear stem cells at protracted postmortem intervals is probably more challenging than the isolation of inner ear stem cells from neonatal mice. Another sobering fact is that, in murine inner ear, stem cells can only be robustly isolated from the vestibular system (Oshima et al. 2007) and just sporadically occurred from cochlear tissues. If these facts are broadly applicable to adult human inner ear tissues, we expect that isolation of stem cells from the vestibular system will be more straightforward than from cochlear tissues. However, a recent study has reported the isolation of sphere forming stem cells from the spiral ganglion of adult humans and guinea pigs (Rask-Andersen et al. 2005), which might point to an inherent difference between mice and humans or guinea pigs. Alternatively, this difference might be caused by procedural differences in cell isolation and/or handling. Our own observations indicate that 
small procedural changes have substantial impact on the efficiency of stem cell isolation, which lead us to expect that future studies will improve on the isolation procedures of different types of inner ear stem cells. We are therefore optimistic that it will be possible to routinely isolate stem cells from adult human vestibular tissue and possibly also from cochlear tissues.

\section{ACKNOWLEDGEMENTS}

The authors thank the members of our group, particularly Anthony Peng and Eva Clamann, for valuable discussions and for suggestions on the manuscript. Begun at the Massachusetts Eye \& Ear Infirmary, this work was supported in part by grants PBBEB-105075 (2005) and 1226/PASMA-111607/1 (2006) from the Swiss National Science Foundation to P.S., by a McKnight Endowment Fund for Neuroscience Brain Disorders Award and grants from the National Institutes of Health DC006167 and DC007132 to S.H.

\section{REFERENCES}

Fujino K, Kim TS, Nishida AT, Nakagawa T, Omori K, Naito Y, Ito J. Transplantation of neural stem cells into explants of rat inner ear. Acta Otolaryngol. Suppl. 124:31-33, 2004.

Heller S, Bell AM, Denis CS, Choe Y, Hudspeth AJ. Parvalbumin 3 is an abundant $\mathrm{Ca} 2+$ buffer in hair cells. J. Assoc. Res. Otolaryngol. 3:488-498, 2002.

LAYwell ED, KuKeKov VG, Steindler DA. Multipotent neurospheres can be derived from forebrain subependymal zone and spinal cord of adult mice after protracted postmortem intervals. Exp. Neurol. 156:430-433, 1999.

Li H, Corrales CE, Edge A, Heller S. Stem cells as therapy for hearing loss. Trends Mol. Med. 10:309-315, 2004a.

Li H, Liu H, Balt S, Mann S, Corrales Ce, Heller S. Correlation of expression of the actin filament-bundling protein espin with stereociliary bundle formation in the developing inner ear. J. Comp. Neurol. 468:125-134, 2004b.

Li H, Liu H, Heller S. Pluripotent stem cells from the adult mouse inner ear. Nat. Med. 9:1293-1299, 2003a.
Li H, Roblin G, Liu H, Heller S. Generation of hair cells by stepwise differentiation of embryonic stem cells. Proc. Natl. Acad. Sci. USA. 100:13495-13500, 2003b.

Lumpkin EA, Collisson T, Parab P, Omer-Abdalla A, Haeberle H, Chen P, Doetzlhofer A, White P, Groves A, Segil N, Johnson JE. Math1-driven GFP expression in the developing nervous system of transgenic mice. Gene Expr. Patterns 3:389-395, 2003.

Mayer EJ, Carter DA, Ren Y, Hughes EH, Rice CM, Halfpenny CA, Scolding NJ, Dick AD. Neural progenitor cells from postmortem adult human retina. Br. J. Ophthalmol. 89:102-106, 2005.

McKaY R. Stem cells in the central nervous system. Science 276: 66-71, 1997.

Oshima K, Grimm C, Corrales CE, Senn P, Martinez Monedero R, Geleoc GS, Edge A, Holt JC, Heller S. Differential distribution of stem cells in the auditory and vestibular organs of the inner ear. J. Assoc. Res. Otolaryngol. 8(1):18-31, 2007, DOI: 10.1007/ s10162-006-0058-3.

Palmer TD, Schwartz PH, Taupin P, Kaspar B, Stein SA, Gage FH. Cell culture. Progenitor cells from human brain after death. Nature 411:42-43, 2001.

Parker MA, Cotanche DA. The potential use of stem cells for cochlear repair. Audiol. Neurootol. 9:72-80, 2004.

Pritchett K, Corrow D, Stockwell J, Sмith A. Euthanasia of neonatal mice with carbon dioxide. Comp. Med. 55:275-81, 2005.

Rask-Andersen H, Bostrom M, Gerdin B, Kinnefors A, Nyberg G, Engstrand T, Miller JM, Lindholm D. Regeneration of human auditory nerve. In vitro/in video demonstration of neural progenitor cells in adult human and guinea pig spiral ganglion. Hear. Res. 203:180-191, 2005.

Roblin G, Li H, Heller S. Expression of the Hair Cell Markers Espin and Parvalbumin 3 During Mouse and Chicken Inner Ear Development. Association for Research in Otolaryngology MidWinter Meeting, Daytona Beach, FL, pp. 359, 2003.

Roisen FJ, Klueber KM, Lu CL, Hatcher LM, Dozier A, Shields CB, MAguire S. Adult human olfactory stem cells. Brain Res. 890: 11-22, 2001.

Rosenquist MD, Kealey GP, Lewis RW, Cram AE. A comparison of storage viability of nonmeshed and meshed skin at 4 degrees C. J. Burn Care Rehabil. 9:634-636, 1988.

Tateya I, Nakagawa T, Iguchi F, Kim TS, Endo T, Yamada S, Kageyama R, Naгto Y, Ito J. Fate of neural stem cells grafted into injured inner ears of mice. Neuroreport 14:1677-1681, 2003.

Zheng L, Sekerkova G, Vranich K, Tilney LG, Mugnaini E, Bartles $\mathrm{JR}$. The deaf jerker mouse has a mutation in the gene encoding the espin actin-bundling proteins of hair cell stereocilia and lacks espins. Cell 102:377-385, 2000. 